\title{
Tuning the Refractive Index and Optical Band Gap of Silk Fibroin Films by Electron Irradiation
}

\author{
S. Asha, ${ }^{1}$ Y. Sangappa, ${ }^{1,2}$ and Sanjeev Ganesh ${ }^{1}$ \\ ${ }^{1}$ Department of Studies in Physics, Mangalore University, Mangalagangotri, Mangalore 574 199, India \\ ${ }^{2}$ School of Materials Science and Engineering, Georgia Institute of Technology, Atlanta, GA 30332, USA \\ Correspondence should be addressed to S. Asha; a_shinda@yahoo.co.in
}

Received 2 March 2015; Revised 8 April 2015; Accepted 12 April 2015

Academic Editor: Austin Nevin

Copyright (c) $2015 \mathrm{~S}$. Asha et al. This is an open access article distributed under the Creative Commons Attribution License, which permits unrestricted use, distribution, and reproduction in any medium, provided the original work is properly cited.

\begin{abstract}
The Bombyx mori silk fibroin (SF) films were prepared by solution casting method and effects of electron beam on the optical properties and optical constants of the films have been studied by using UV-Visible spectrophotometer. Optical properties like optical band gap $E_{g}$, refractive index $n$, extinction coefficient $k$, optical conductivity $\sigma_{\text {opt }}$, and dielectric constants $\varepsilon^{*}$ of virgin and electron irradiated films were determined by using UV-Visible absorption and transmission spectra. It was found that the reduction in optical band gap and increase in refractive index with increasing radiation dosage was observed. It is also observed from results that there is increase in dielectric constants with increasing photon energy. The observed optical changes have been tried to be correlated with the structural changes, revealed through FT-IR spectroscopy. The present study is quite important for tailoring the optical responses of SF films as per specific requirements.
\end{abstract}

\section{Introduction}

Silk is a natural semicrystalline biopolymer. Bombyx mori silk consists of two kinds of proteins, fibroin and sericin [1]. Silk fibroin (SF) can be obtained as pure regenerated solution from the silk fibers of Bombyx mori silkworm, by water based process. Silk fibroin from the Bombyx mori silkworm is easily formed into a variety of material forms such as hydrogels [2], ultrathin and thick films $[3,4]$, controlled-release coatings [5], and 3D porous matrices [6].

SF films are mechanically robust having very smooth surfaces and highly transparent across the visible region and possess high refractive index [7,8]. Silk fibroin (SF) is found to be well suited for optical applications in film form with free standing materials of thickness between 20 and $100 \mu \mathrm{m}$. This is possible due to regenerated silk fibroin in the solid state containing extremely stable $\beta$-pleated sheets. These secondary structures are stabilized by interchain hydrogen bonding in which every amino acid is laterally bonded twice to its nearest neighbor chain segments. The hydrogen bonds are responsible for the extraordinary physical properties like high tensile strength, toughness, biocompatibility, and biodegradability $[9,10]$. SF has been used for centuries for biomedical applications, but it is receiving increasing attention as technological material. Silk fibroin in film form has been recognized as a suitable biopolymer for the development of optical devices [11], optical waveguides [12], luminescent solar concentrators [13], 2D, 3D optical elements-holograms, lenses, optical gratings, and antireflective coatings $[14,15]$.

Nowadays the irradiation of polymers is one of the important fields for altering the polymer properties like electrical, optical, chemical, mechanical, and so forth, significantly [16]. The interaction of radiation with polymers leads to chain scission, chain aggregation, formation of double bonds, and molecular emission. As a result of this the polymer properties may be modified [17]. The knowledge of optical properties of silk is essential as it is used as biosensors, antireflective coatings, solar cells, optical waveguides, and so forth. This justifies the need for studying the irradiation effects on the optical properties of the SF fibroin films such as absorption, extinction coefficients, and optical parameters like energy band gap, refractive index, and optical conductivity. We have reported the influence of $8 \mathrm{MeV}$ electron irradiation effects on the optical properties and optical constants of silk fibroin films (silk taken from Mysore, India). 
TABLE 1: Specifications of the electron beam accelerator and irradiation conditions.

\begin{tabular}{lc}
\hline (1) Beam energy & $8 \mathrm{MeV}$ \\
(2) Beam current & $20 \mathrm{~mA}$ \\
(3) Pulse repetition rate & $50 \mathrm{~Hz}$ \\
(4) Pulse width & $2.2 \mu \mathrm{s}$ \\
(5) Distance source to sample & $30 \mathrm{~cm}$ \\
(6) Dose range & $0-150 \mathrm{kGy}$ \\
(7) Atmosphere & $\mathrm{Air}$ \\
(8) Temperature & $24^{\circ} \mathrm{C}$ \\
\hline
\end{tabular}

\section{Experimental}

2.1. Materials and Methods. The Bombyx mori CSR4 cocoons were obtained from silkworms reared in controlled condition at the Sericulture Department, University of Mysore, India. $\mathrm{Na}_{2} \mathrm{CO}_{3}$ and $\mathrm{LiBr}$ (>99\% purity, Sigma Aldrich) were used without purification. Bombyx mori CSR4 silk cocoons were cut into small pieces and the product was rinsed with water, then treated with boiling aqueous solution of $0.02 \mathrm{M}$ $\mathrm{Na}_{2} \mathrm{CO}_{3}$ for $40 \mathrm{~min}$. with stirring, then rinsed thoroughly with distilled water to remove the glue like sericin protein, and then dried in hot air oven. The silk fibroin solution was prepared by dissolving $6 \mathrm{~g}$ of degummed silk in $9.3 \mathrm{M} \mathrm{LiBr}$ solution at $60^{\circ} \mathrm{C}$ for $4 \mathrm{~h}$. This solution was dialyzed against distilled water using a dialysis cassette with molecular, cutoff weight $3500 \mathrm{Da}$ for $72 \mathrm{~h}$ changing water every $6 \mathrm{~h}$ in order to remove salt. This solution was optically clear after dialysis and was centrifuged to remove the small amount of silk aggregates that formed during the process. The final concentration of SF was about $1 \%(\mathrm{w} / \mathrm{v})$. The prepared fibroin solution was transferred into a polystyrene Petri dish, allowing the solvent to evaporate at room temperature for 3 days before the films were peeled off from the plates. The final films were vacuum dried and stored in desiccators to avoid the absorption of moisture. The thickness of the obtained films was $60 \pm 0.8 \mu \mathrm{m}$. The transparency of the SF films in the visible range was more than $90 \%$.

2.2. Electron Irradiation. The polymers samples of size $(2 \times$ 2) $\mathrm{cm}^{2}$ were cut from the prepared films. One sample was kept as virgin and the other three samples were subjected to irradiation of different doses. Irradiation of samples was carried out at Microtron Center, Mangalore University, India, using the electron beam (by lanthanum hexafluorite source). The features of monochromatic beam are given in Table 1 .

2.3. UV-Visible Spectroscopy. The UV-Visible absorption spectra of virgin and high energy electron irradiated silk fibroin film samples have been studied by using UV-Visible spectrophotometer (Shimadzu UV-1800, Japan) in the wavelength range $190-800 \mathrm{~nm}$. All the spectra were recorded by mounting the samples in the integrating sphere assembly attached to the spectrometer, keeping air as the reference.

2.4. Fourier Transforms Infrared Spectra. Fourier transform infrared (FT-IR) spectra of the samples were recorded in

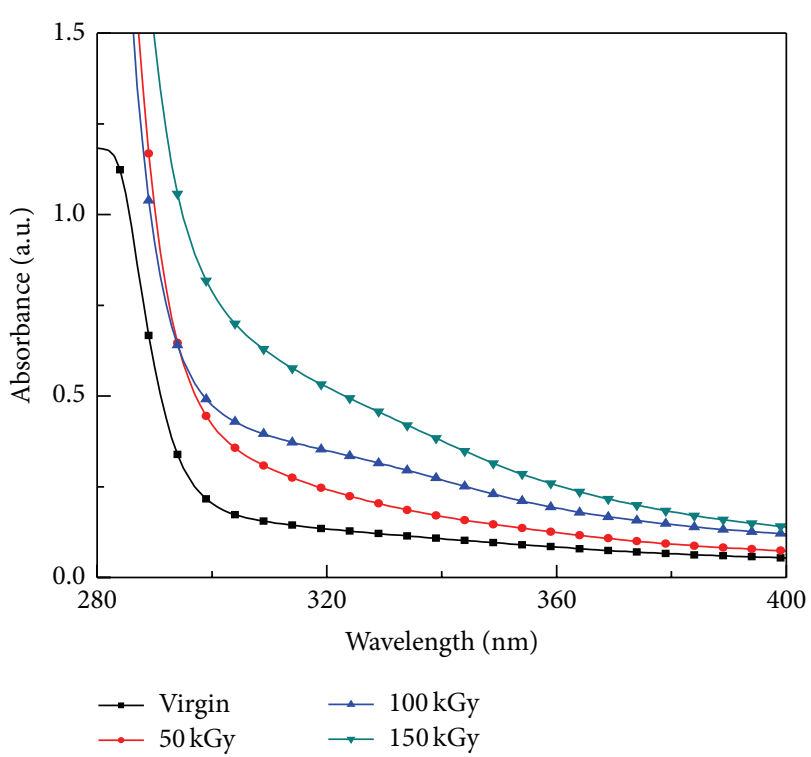

FIGURE 1: Absorption spectra of virgin and electron irradiated silk fibroin films.

transmission mode using Thermo Nicolet, Avatar 370, FTIR spectrophotometer having a resolution $4 \mathrm{~cm}^{-1}$ in the wavenumber range $500-4000 \mathrm{~cm}^{-1}$.

\section{Experimental Results and Discussion}

3.1. Study of Optical Behaviour. The transition of electrons in different orbitals from the ground state to excited energy states occurs during the absorption of light energy by polymeric materials in the ultraviolet and visible regions. The electronic transitions involved the following types: $\sigma \rightarrow \sigma^{*}$, $\mathrm{n} \rightarrow \sigma^{*}, \mathrm{n} \rightarrow \pi^{*}$, and $\pi \rightarrow \pi^{*}$. Many of the optical transitions which result from the presence of impurities have energies in the visible part of the spectrum. Consequently the defects are referred to as colour centres [18]. Energetic beam interaction with polymers generates damage which leads to the formation of new defects and new charge states. Ultraviolet-Visible (UV-Vis) spectrophotometer has become an important tool for investigation of these electronic transitions. It is used to estimate the value of optical energy band gap $\left(E_{g}\right)$ in polymers.

The UV-Visible absorption spectra of virgin and $8 \mathrm{MeV}$ electron irradiated (50-150 kGy) silk fibroin polymer samples are presented in Figure 1, in the wavelength range 280$400 \mathrm{~nm}$. The recorded spectra of virgin silk fibroin film display absorption at $300 \mathrm{~nm}$ which is assigned to $\pi \rightarrow$ $\pi^{*}$ transition of the Tyr, Phe, and Try residues in the SF molecular chain [19]. The intensity of the absorption band changes with increasing dose, while its position slightly shifted towards the higher wavelength side [20]. This may be because electron irradiation of silk fibroin causes the creation of carbonyl group. This lowers the energy required for the optical transition so the absorption moves towards longer wavelengths. The carbonyl group modifies the optical transitions and hence the optical band gap. 
TABLE 2: Band gap energy and Urbach energy of virgin and electron irradiated silk fibroin films.

\begin{tabular}{lcc}
\hline Sample & Band gap energy $(\mathrm{eV})$ & Urbach energy $(\mathrm{eV})$ \\
\hline Virgin & 4.18 & 0.524 \\
$50 \mathrm{kGy}$ & 4.12 & 0.452 \\
$100 \mathrm{kGy}$ & 4.08 & 0.430 \\
$150 \mathrm{kGy}$ & 3.94 & 0.426 \\
\hline
\end{tabular}

3.1.1. Absorption Coefficient and Optical Band Gap. The electron irradiation of polymer causes change in the polymer structure; hence a modification within the optical band gap is expected. The information about the optical band gap is obtained from the absorption edge of the UV-Vis spectra. Practically the optical absorption coefficient $\alpha$ can be calculated from the optical absorbance $A$, by using relation [18]

$$
\alpha(h v)=\frac{2.303 A}{X},
$$

where $X$ is the sample thickness in $\mathrm{cm}$ and $A$ is defined as $A=$ $\log \left(I_{0} / I\right)$, where $I_{0}$ and $I$ are the intensity of the incident and transmitted beam, respectively. Here the optical band gap was determined from the analysis of the spectral dependence of the optical absorption near the absorption edge. The optical absorption coefficient for noncrystalline materials has the following frequency dependence [21-23]:

$$
\alpha(h v)=\frac{B\left(h v-E_{g}\right)^{r}}{h v},
$$

where $h v$ is the energy of the incident photons, $E_{g}$ is the optical energy gap between the valence band and conduction band, and $r$ is an empirical exponent which characterizes the type of optical transition during the absorption process. The exponent $r$ has value of $1 / 2$ for allowed direct transition, while it takes the value $3 / 2$ for the direct forbidden transition. And it has values 2, 3 for indirect allowed and forbidden transitions, respectively [24]. The simplest way to obtain the type of transition is to examine the value of $r$, which fits $h \nu$ to $\alpha h v$ with a straight line relationship. In our case, $r=$ $1 / 2$ which means an allowed direct transition, where the electron transition is vertical from the top of the valence band to the bottom of the conduction band. The nonvertical transitions are forbidden in this case. The factor $B$ depends on the transition probability and can be assumed to be constant within the optical frequency range. The usual method for the determination of the value of $E_{g}$ involves plotting $(\alpha h \nu)^{2}$ against $(h \nu)$. Figure 2 illustrates $(\alpha h \nu)^{2}$ as a function of the photon energy $h v$ for virgin and electron irradiated films. The values of the optical band gap $E_{g}$ are normally obtained by extrapolation of the linear part of the curve to intersect the $x$-axis at zero absorption [25] which gives the corresponding energy band gap and which is presented in Table 2.

3.1.2. Urbach Energy. The exponential dependence on the photon energy $(h \nu)$ by the absorption coefficient near

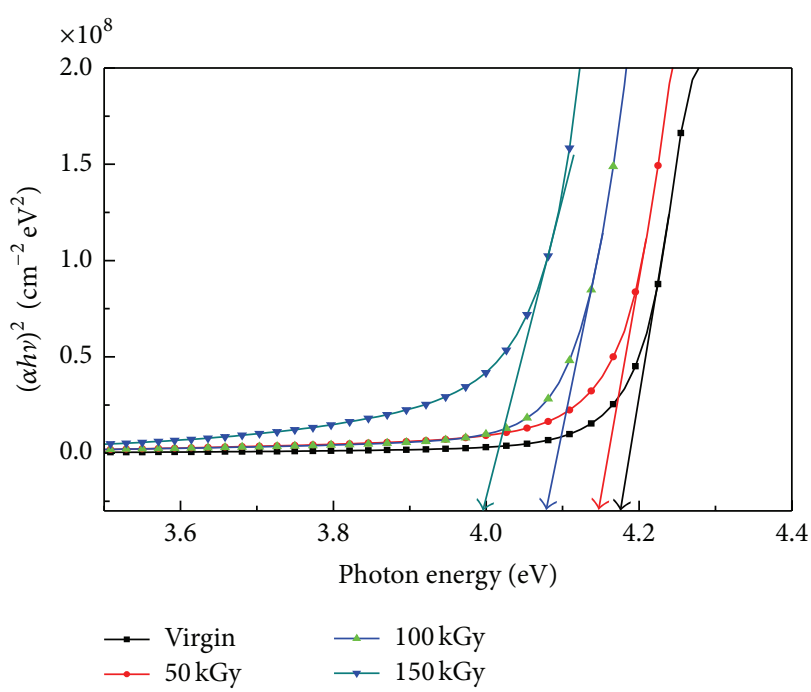

FIGURE 2: Variation of $(\alpha h \nu)^{2}$ as a function of the photon energy $h \nu$.

the band edge for noncrystalline materials follows the Urbach relation $[26,27]$

$$
\alpha(\nu)=\alpha_{0} \exp \left(\frac{h v}{E_{u}}\right)
$$

where $\alpha_{0}$ is a constant, $E_{u}$ is an energy which is interpreted as the width of the tail of localized states in the forbidden band gap, $v$ is the frequency of radiation, and $h$ is Planck's constant. Figure 3 shows the variation in the logarithm of the absorption coefficient as a function of the photon energy for all unirradiated and electron irradiated SF samples. The value of Urbach energy $\left(E_{u}\right)$ is normally calculated by considering the reciprocal of the slope of the linear portion in the lower photon energy region of these curves. The calculated values of Urbach energy for virgin and electron irradiated silk fibroin film samples are presented in Table 2. The decrease in Urbach's energy may be due to the decrease in the crystalline nature of the polymer [28]. Upon irradiation, the transparent silk fibroin film polymer became yellow and the degree of yellowness was found to be increased with increasing electron irradiation dose. Treatment of silk with radiations is reported to produce yellow colour due to oxidation of amino acid residues glycine, alanine, serine, and tyrosine $[29,30]$ and especially formation of chromophore products $[31,32]$.

3.1.3. Determination of Refractive Index. For many devices, accurate knowledge of the refractive index and its frequency dependence is very important. The refractive index $(n)$ values for virgin and high energy electron irradiated SF samples at different wavelengths have been calculated by using the relation [27]:

$$
n=\left\{\left[\frac{4 R}{(R-1)^{2}}-k^{2}\right]^{1 / 2}-\frac{R+1}{R-1}\right\},
$$

where $k$ is the extinction coefficient. The extinction coefficient is a measure of the fraction of light lost due to scattering 


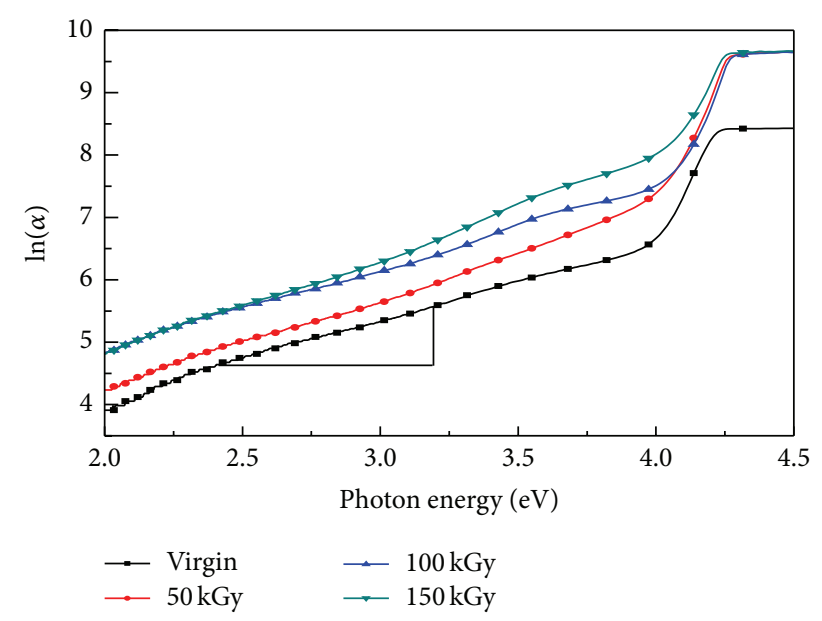

FIGURE 3: Urbach energy for virgin and electron irradiated silk fibroin films.

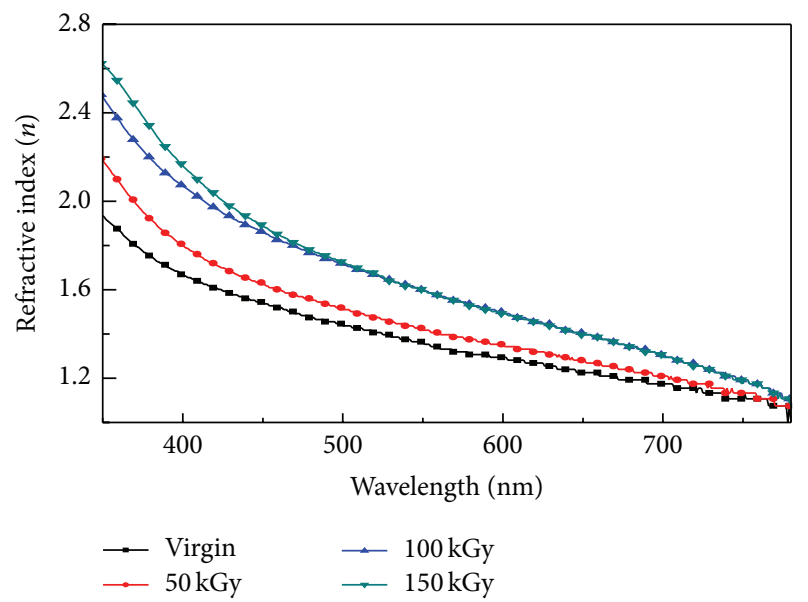

FIGURE 4: Variation of refractive index as a function of wavelength.

and absorption per unit distance of the penetration medium. It can be estimated from the values of $\alpha$ and $\lambda$ using the relation [33]

$$
k=\frac{\alpha \lambda}{4 \pi} .
$$

The spectral distributions of the values of $n$ and $k$ for different doses are shown in Figures 4 and 5, respectively.

Figure 4 shows the refractive index $n$ increases with irradiation doses; indeed $n$ changes from 1.72 to 2.13 at $600 \mathrm{~nm}$, when radiation dose increases from 0 to $150 \mathrm{kGy}$. It is observed that the refractive index increased with increasing dosage and the radiation induced refractive index changes are very large. These changes showed that exposure of SF films to energetic electron radiation is most acceptable approach to tailor the refractive index efficiently. It has a potential applicability for optical devices, for example, optical waveguides and photonic devices $[34,35]$. The dependence of extinction coefficient $k$ on the wavelength is presented in Figure 5 for virgin and electron irradiated samples.

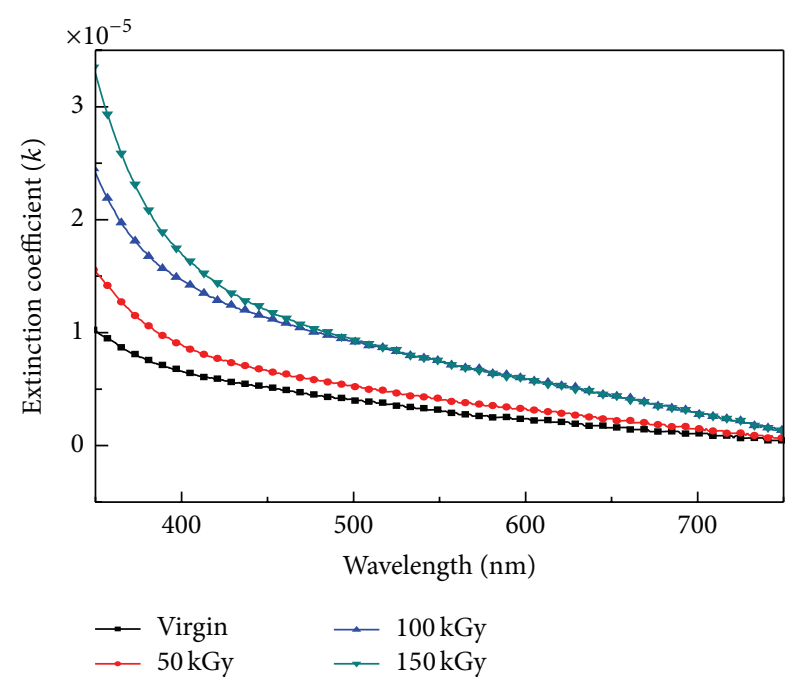

FIGURE 5: Variation of extinction coefficient $k$ as a function of wavelength.

The extinction coefficient $k$ shows clear changes with increasing doses; indeed, $k$ changes from $1.3 \times 10^{-6}$ to $2.5 \times 10^{-6}$ at $\lambda=630 \mathrm{~nm}$ for increase in radiation dose from 0 to $150 \mathrm{kGy}$. This change in refractive index and extinction coefficient with increasing irradiation is attributed to structural modifications occurring in the samples.

3.1.4. Optical Conductivity. Using the absorption coefficient $(\alpha)$ and refractive index $(n)$ of the material one can calculate the optical responses of a material in terms of the optical conductivity $\sigma_{\text {opt }}$, which is given by the relation $[36,37]$

$$
\sigma_{\mathrm{opt}}=\frac{\alpha n c}{4 \pi}
$$

where $c$ is the velocity of light.

Figure 6 shows the variation of the optical conductivity as a function of photon energy for the SF samples. From Figure 6 it is very clear that the optical conductivity increases with increase in photon energy and also increasing radiation doses under investigation. The increase in optical conductivity can be attributed to the increase in absorption coefficient.

3.1.5. Determination of Dielectric Properties. The complex dielectric constant $\left(\varepsilon^{*}=\varepsilon_{r}+i \varepsilon_{i}\right)$ is a fundamental property of the material, which characterizes the optical properties of a solid material. The dielectric constant consists of two components, real part and imaginary part. The real part of the dielectric constant shows how much it will slow down the speed of light in the material, whereas the imaginary part indicates how a dielectric material absorbs energy from an electric field due to dipole motion. The knowledge of the real and imaginary parts of the dielectric constant gives the information about the loss factor which is the ratio of the imaginary part to the real part of the dielectric constant. 


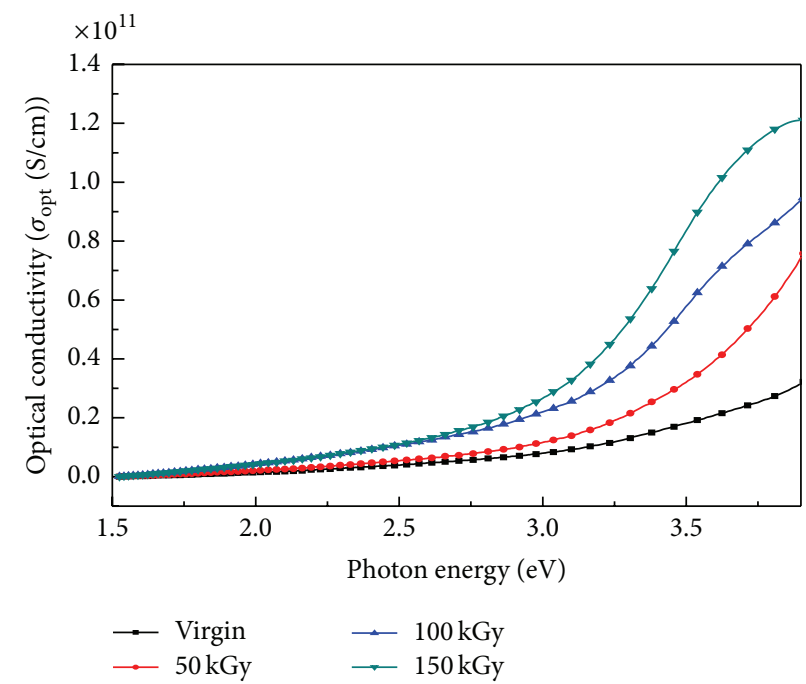

FIGURE 6: Variation of optical conductivity as a function of photon energy.

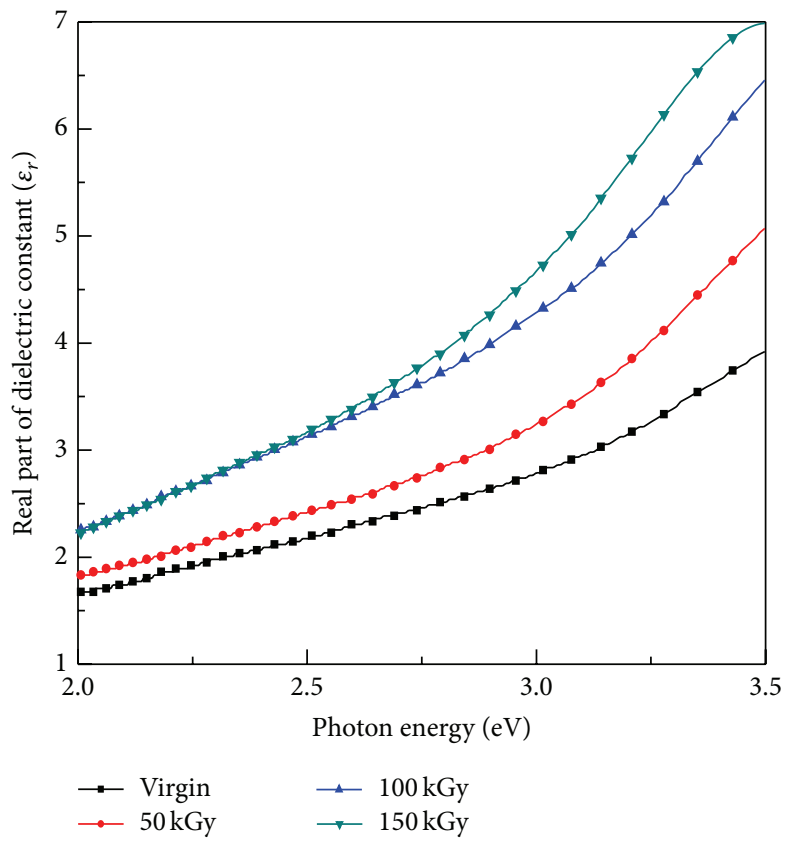

(a)

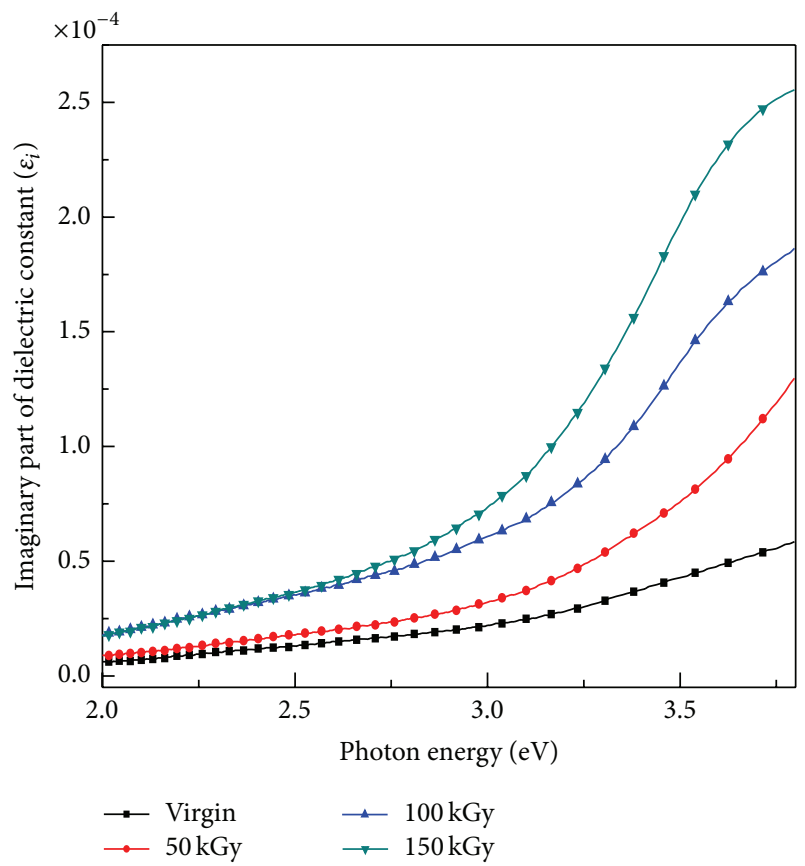

(b)

FIGURE 7: Variation of (a) real part of dielectric constant and (b) imaginary part of dielectric constant as a function of photon energy.

The real and imaginary parts of the dielectric constant can be determined by the following relations [38]:

$$
\begin{aligned}
\varepsilon_{r} & =n^{2}-k^{2}, \\
\varepsilon_{i} & =2 n k .
\end{aligned}
$$

Figures $7(\mathrm{a})$ and $7(\mathrm{~b})$ show the variation of the real and imaginary parts of the dielectric constant as a function of photon energy for the virgin and electron irradiated SF samples. From the figures it is clear that both real and imaginary parts of the dielectric constant increase with increase in photon energy. The real part of the dielectric constant depends on refractive index by (7) because the effect of extinction coefficient is very small and hence has a negligible contribution [39]. Increase in real part was observed with increase in photon energy and irradiation doses. The imaginary part of the dielectric constant depends on extinction coefficient by (8) because the refractive index is very small [40]. From the figure it is clear that the value of imaginary dielectric constant increases slowly in the lower energy region and increases sharply in the higher energy region. These results indicate that the loss factor increases with increase in photon energy. 


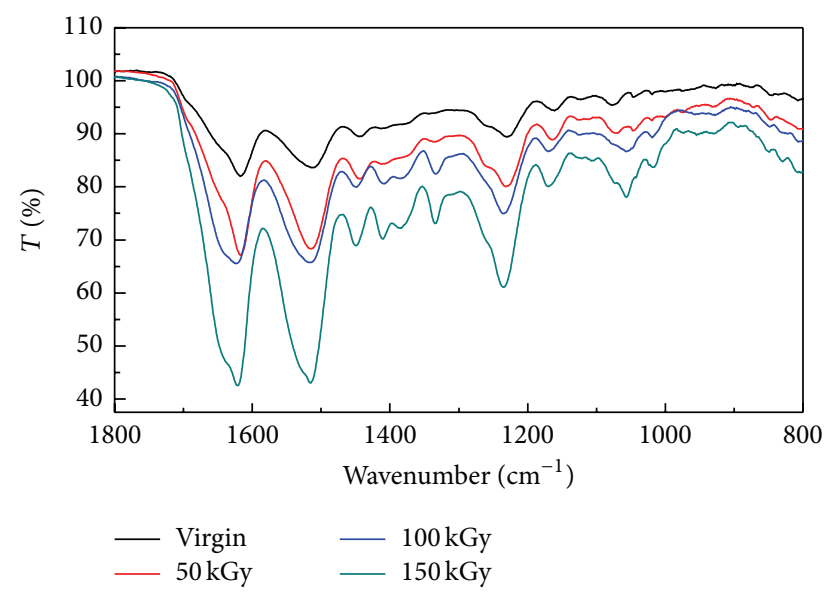

FIGURE 8: FT-IR spectra of virgin and electron irradiated silk fibroin films.

\subsection{Structural Studies}

3.2.1. FT-IR Analysis. The structure of fibroin proteins in the native SF and electron irradiated films were characterized by FT-IR spectroscopy. The FT-IR spectra of the native $\mathrm{SF}$ and electron irradiated films are given in Figure 8. The position of absorption bands indicated the conformation of fibroin protein. The absorption bands observed for SF are at $1617 \mathrm{~cm}^{-1}$ (amide I) which is mainly related with the $\mathrm{C}=\mathrm{O}$ stretching, $1511 \mathrm{~cm}^{-1}$ (amide II) $\mathrm{C}-\mathrm{H}$ stretching vibration, and $1236 \mathrm{~cm}^{-1}$ (amide III) $\mathrm{C}-\mathrm{N}$ stretching vibration. In addition, the position of the amide bands indicates the conformations of the fibroin protein components, $1620 \mathrm{~cm}^{-1}$ ( $\beta$ sheet) for amide $\mathrm{I}, 1520 \mathrm{~cm}^{-1}$ ( $\beta$ sheet) for amide II, and $1230 \mathrm{~cm}^{-1}$ (random coil), were assigned as both silk I and silk II structure $[41,42]$ of the SF. This indicates that both random coil ( $\alpha$-helices) and $\beta$ sheet structure occur in SF simultaneously. When the SF films were subjected to high energy electron irradiation, the FT-IR results showed that the absorption bands 1617,1511 , and $1229 \mathrm{~cm}^{-1}$ were shifted to 1623,1516 , and $1236 \mathrm{~cm}^{-1}$, respectively. Also the FT-IR spectrum shows the intensity of amides I-III, increased as irradiation dose increased, indicates the weakening of the peptide bond $(\mathrm{C}-\mathrm{N})$ in polypeptides [43]. Such structural changes as revealed through FT-IR spectroscopy corroborate the observations from optical measurements.

\section{Conclusions}

The basic optical properties and optical constants of the unirradiated and electron beam irradiated SF films have been investigated by means of absorbance and transmittance spectra. The observed variation in optical parameters such as the optical band gap $\left(E_{g}\right)$, refractive index $(n)$, extinction coefficient $(k)$, optical conductivity $\left(\sigma_{\text {opt }}\right)$, and dielectric constants $\left(\varepsilon^{*}\right)$ of SF may lead to tailoring of the optical properties of this biopolymer as per specific requirements after electron irradiation. Refractive index and optical conductivity of the polymer increase with increasing radiation dosage indicating
SF films are suitable for antireflective coating, photonic devices, and image sensors. To our knowledge, this is the first systematic work to report the variation in the refractive index of SF, in the almost complete visible range, as a result of electron irradiation.

\section{Conflict of Interests}

The authors declare that there is no conflict of interests regarding the publication of this paper.

\section{Acknowledgment}

The first author (S. Asha) thanks UGC (New Delhi, India) for an RGNF fellowship.

\section{References}

[1] A. D. Metcalfe and M. W. J. Ferguson, "Bioengineering skin using mechanisms of regeneration and repair," Biomaterials, vol. 28, no. 34, pp. 5100-5113, 2007.

[2] U.-J. Kim, J. Park, C. Li, H.-J. Jin, R. Valluzzi, and D. L. Kaplan, "Structure and properties of silk hydrogels," Biomacromolecules, vol. 5, no. 3, pp. 786-792, 2004.

[3] C. Jiang, X. Wang, R. Gunawidjaja et al., "Mechanical properties of robust ultrathin silk fibroin films," Advanced Functional Materials, vol. 17, no. 13, pp. 2229-2237, 2007.

[4] X. Y. Wang, H. J. Kim, P. Xu, A. Matsumoto, and D. L. Kaplan, "Biomaterial coatings by stepwise deposition of silk fibroin," Langmuir, vol. 21, no. 24, pp. 11335-11341, 2005.

[5] X. Wang, X. Hu, A. Daley, O. Rabotyagova, P. Cebe, and D. L. Kaplan, "Nanolayer biomaterial coatings of silk fibroin for controlled release," Journal of Controlled Release, vol. 121, no. 3, pp. 190-199, 2007.

[6] S. Ghosh, S. T. Parker, X. Wang, D. L. Kaplan, and J. A. Lewis, "Direct-write assembly of microperiodic silk fibroin scaffolds for tissue engineering applications," Advanced Functional Materials, vol. 18, no. 13, pp. 1883-1889, 2008.

[7] G. H. Altman, F. Diaz, C. Jakuba et al., "Silk-based biomaterials," Biomaterials, vol. 24, no. 3, pp. 401-416, 2003.

[8] B. D. Lawrence, F. Omenetto, K. Chui, and D. L. Kaplan, "Processing methods to control silk fibroin film biomaterial features," Journal of Materials Science, vol. 43, no. 21, pp. 69676985, 2008.

[9] Z. Shao and F. Vollrath, "Surprising strength of silkworm silk," Nature, vol. 418, no. 6899, article 741, 2002.

[10] C. Holland, F. Vollrath, A. J. Ryan, and O. O. Mykhaylyk, "Silk and synthetic polymers: reconciling 100 degrees of separation," Advanced Materials, vol. 24, no. 1, pp. 105-109, 2012.

[11] D. H. Kim, Y. S. Kim, J. Amsden et al., "Silicon electronics on silk as a path to bioresorbable, implantable devices," Applied Physics Letters, vol. 95, Article ID 133701, 2009.

[12] S. T. Parker, P. Domachuk, J. Amsden et al., "Biocompatible silk printed optical waveguides," Advanced Materials, vol. 21, no. 23, pp. 2411-2415, 2009.

[13] Y. Tsuboi, H. Adachi, K. Yamada, H. Miyasaka, and A. Itaya, "Laser ablation of silk protein (fibroin) films," Japanese Journal of Applied Physics, vol. 41, no. 7, pp. 4772-4779, 2002.

[14] B. D. Lawrence, M. Cronin-Golomb, I. Georgakoudi, D. L. Kaplan, and F. G. Omenetto, "Bioactive silk protein biomaterial 
systems for optical devices," Biomacromolecules, vol. 9, no. 4, pp. 1214-1220, 2008.

[15] H. Perry, A. Gopinath, D. L. Kaplan, L. D. Negro, and F. G. Omenetto, "Nano- and micropatterning of optically transparent, mechanically robust, biocompatible silk fibroin films," Advanced Materials, vol. 20, no. 16, pp. 3070-3072, 2008.

[16] D. Sinha, K. L. Sahoo, U. B. Sinha, T. Swu, A. Chemseddine, and D. Fink, "Gamma-induced modifications of polycarbonate polymer," Radiation Effects and Defects in Solids, vol. 159, no. 10, pp. 587-595, 2004.

[17] S. V. Kumar, B. Ghadei, P. K. Jal, K. Dey, J. B. M. Krishna, and A. Saha, "Modification of polyethersulphone induced by high energy proton, $\mathrm{C}^{+}$, and $\mathrm{Ne}^{6+}$ ions: a spectroscopic study," Journal of Applied Polymer Science, vol. 101, no. 3, pp. 1591-1597, 2006.

[18] Sangappa, S. Asha, T. Demappa, P. Parameswara, G. Sanjeev, and R. Somashekar, "Spectroscopic and thermal studies of $8 \mathrm{MeV}$ electron beam irradiated HPMC films," Nuclear Instruments and Methods in Physics Research Section B: Beam Interactions with Materials and Atoms, vol. 267, no. 14, pp. 2385-2389, 2009.

[19] M. F. Zaki, "Gamma-induced modification on optical band gap of CR-39 SSNTD," Journal of Physics D: Applied Physics, vol. 41, no. 17, Article ID 175404, 2008.

[20] S. Baltova, V. Vassileva, and E. Valtcheva, "Photochemical behaviour of natural silk-I. Kinetic investigation of photoyellowing," Polymer Degradation and Stability, vol. 60, no. 1, pp. 53-60, 1998.

[21] H.-L. Guo, D.-Y. Liu, X.-D. Yu, and X.-H. Xia, "Direct electrochemistry and electrocatalysis of hemoglobin on nanostructured gold colloid-silk fibroin modified glassy carbon electrode," Sensors and Actuators B: Chemical, vol. 139, no. 2, pp. 598-603, 2009.

[22] H. M. Zidan, "Electron spin resonance and ultraviolet spectral analysis of UV-irradiated PVA films filled with $\mathrm{MnCl}_{2}$ and $\mathrm{CrF}_{3}$," Journal of Applied Polymer Science, vol. 88, no. 1, pp. 104111, 2003.

[23] N. F. Mott and N. F. Devis, Electronic Process in Non-Crystalline Materials, Oxford University Press, New York, NY, USA, 2nd edition, 1979.

[24] J. Tauc, "Optical properties of non-crystalline solids," in Optical Properties of Solid, F. Abeles, Ed., p. 277, North-Holland, Amsterdam, The Netherlands, 1972.

[25] S. Singh and S. Prasher, "The etching and structural studies of gamma irradiated induced effects in CR-39 plastic track recorder," Nuclear Instruments and Methods in Physics Research, Section B, vol. 222, no. 3-4, pp. 518-524, 2004.

[26] F. Urbach, "The long wavelength edge of photographic sensitivity and of the electronic absorption of solids," Physical Review, vol. 92, no. 5, pp. 1324-1326, 1953.

[27] M. D. Migahed and H. M. Zidan, "Influence of UV-irradiation on the structure and optical properties of polycarbonate films," Current Applied Physics, vol. 6, no. 1, pp. 91-96, 2006.

[28] S. Asha, Sangappa, P. Naik, K. S. Chandra, and G. Sanjeev, "Microstructural, thermal and antibacterial properties of electron beam irradiated Bombyx mori silk fibroin films," in 58th DAE Solid State Physics Symposium, vol. 1591 of AIP Conference Proceedings, pp. 219-221, Punjab, India, December 2013.

[29] S. Baltova and V. Vassileva, "Photochemical behaviour of natural silk-II. Mechanism of fibroin photodestruction," Polymer Degradation and Stability, vol. 60, no. 1, pp. 61-65, 1998.
[30] D. Sargunamani and N. Selvakumar, "Effects of ozone treatment on the properties of raw and degummed tassar silk fabrics," Polymer Degradation and Stability, vol. 91, pp. 2644-2653, 2006.

[31] C. Giulivi, N. J. Traaseth, and K. J. A. Davies, "Tyrosine oxidation products: analysis and biological relevance," Amino Acids, vol. 25, no. 3-4, pp. 227-232, 2003.

[32] D. Creed, "The photophysics and photochemistry of the near-uv absorbing amino acids-I. Tryptiphan and its simple derivatives," Photochemistry and Photobiology, vol. 39, no. 4, pp. 537-562, 1984.

[33] A. R. Forouhi and I. Bloomer, "Optical properties of crystalline semiconductors and dielectrics," Physical Review B, vol. 38, no. 3, pp. 1865-1874, 1988.

[34] D.-H. Kim, J. Viventi, J. J. Amsden et al., "Dissolvable films of silk fibroin for ultrathin conformal bio-integrated electronics," Nature Materials, vol. 9, no. 6, pp. 511-517, 2010.

[35] H. Tao, M. A. Brenckle, M. Yang et al., "Silk-based conformal, adhesive, edible food sensors," Advanced Materials, vol. 24, no. 8, pp. 1067-1072, 2012.

[36] N. A. Bakr, A. M. Funde, V. S. Waman et al., "Determination of the optical parameters of a-Si:H thin films deposited by hot wire-chemical vapour deposition technique using transmission spectrum only," Pramana: Journal of Physics, vol. 76, no. 3, pp. 519-531, 2011.

[37] F. Yakuphanoglu, A. Cukurovali, and I. Yilmaz, "Refractive index and optical absorption properties of the complexes of a cyclobutane containing thiazolyl hydrazone ligand," Optical Materials, vol. 27, no. 8, pp. 1363-1368, 2005.

[38] M. A. Omar, Elementary Solid State Physics, Addison-Wesley, New York, NY, USA, 1993.

[39] V. Kumar, P. K. Goyal, S. Mahendia, R. Gupta, T. Sharma, and S. Kumar, "Tuning of the refractive index and optical band gap of CR-39 polymers by heating," Radiation Effects and Defects in Solids, vol. 166, no. 2, pp. 109-113, 2011.

[40] A. H. Ahmad, A. M. Awatif, and N. Z. Abdul-Majied, "Doping effect on optical constants on polymethylmethacrylate (PMMA)," Journal of Engineering \& Technology, vol. 25, no. 4, pp. 558-568, 2007.

[41] M. Tsukada, Y. Gotoh, M. Nagura, N. Minoura, N. Kasai, and G. Freddi, "Structural changes of silk fibroin membranes induced by immersion in methanol aqueous solutions," Journal of Polymer Science Part B: Polymer Physics, vol. 32, no. 5, pp. 961-968, 1994.

[42] A. Kojthung, P. Meesilpa, B. Sudatis, L. Treeratanapiboon, R. Udomsangpetch, and B. Oonkhanond, "Effects of gamma radiation on biodegradation of Bombyx mori silk fibroin," International Biodeterioration and Biodegradation, vol. 62, no. 4, pp. 487-490, 2008.

[43] M. Srisa-Ard, Y. Baimark, and Y. Srisuwan, "Conformation transition and thermal properties study of silk fibroin and poly ( $\varepsilon$-caprolactone) blends," Journal of Applied Sciences, vol. 8, no. 19, pp. 3518-3522, 2008. 

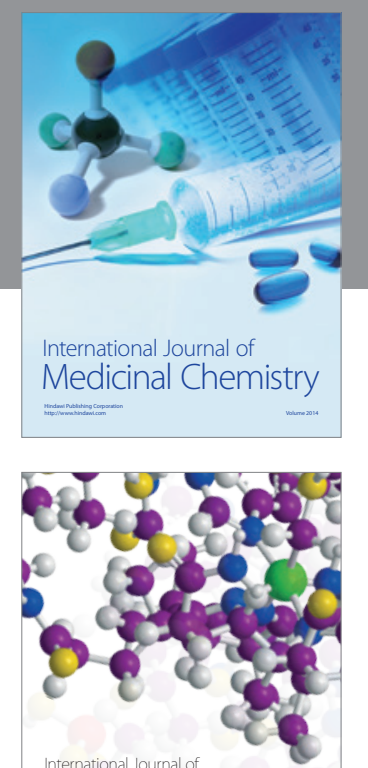

\section{Carbohydrate} Chemistry

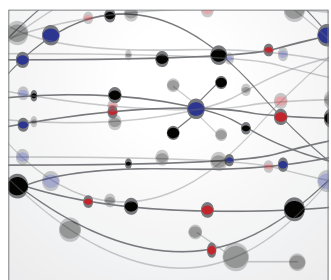

The Scientific World Journal
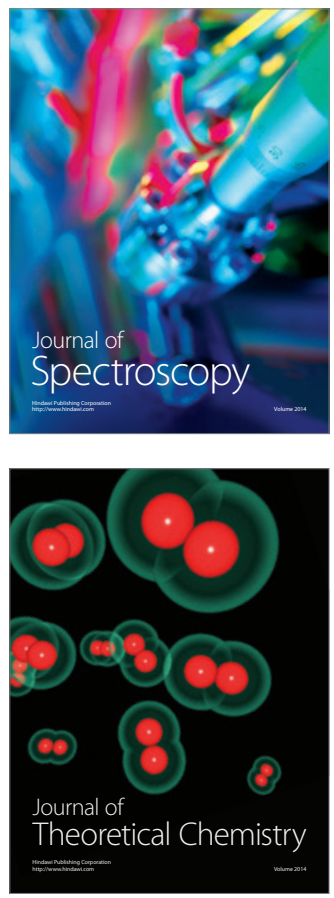
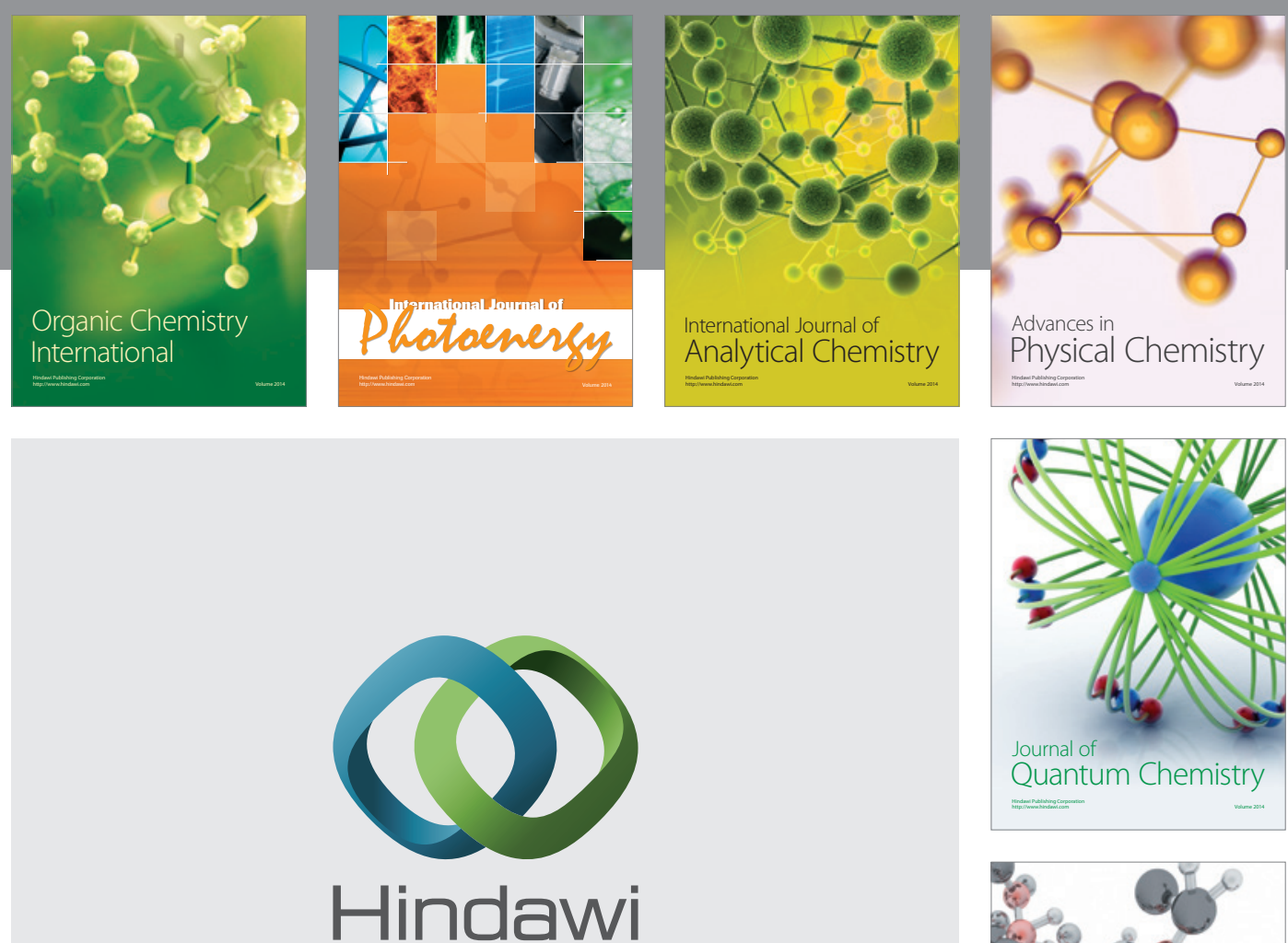

Submit your manuscripts at

http://www.hindawi.com

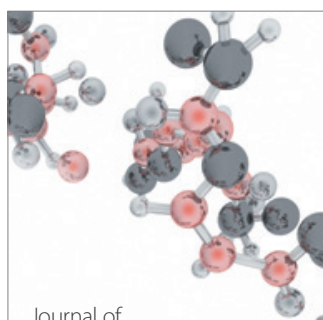

Analytical Methods

in Chemistry

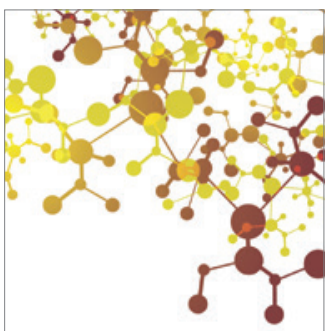

Journal of

Applied Chemistry

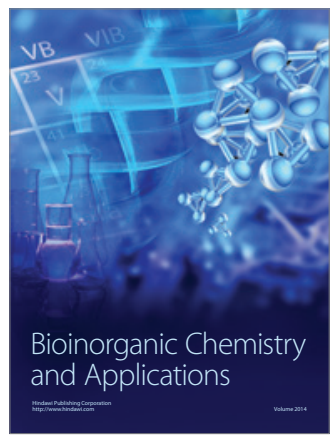

Inorganic Chemistry
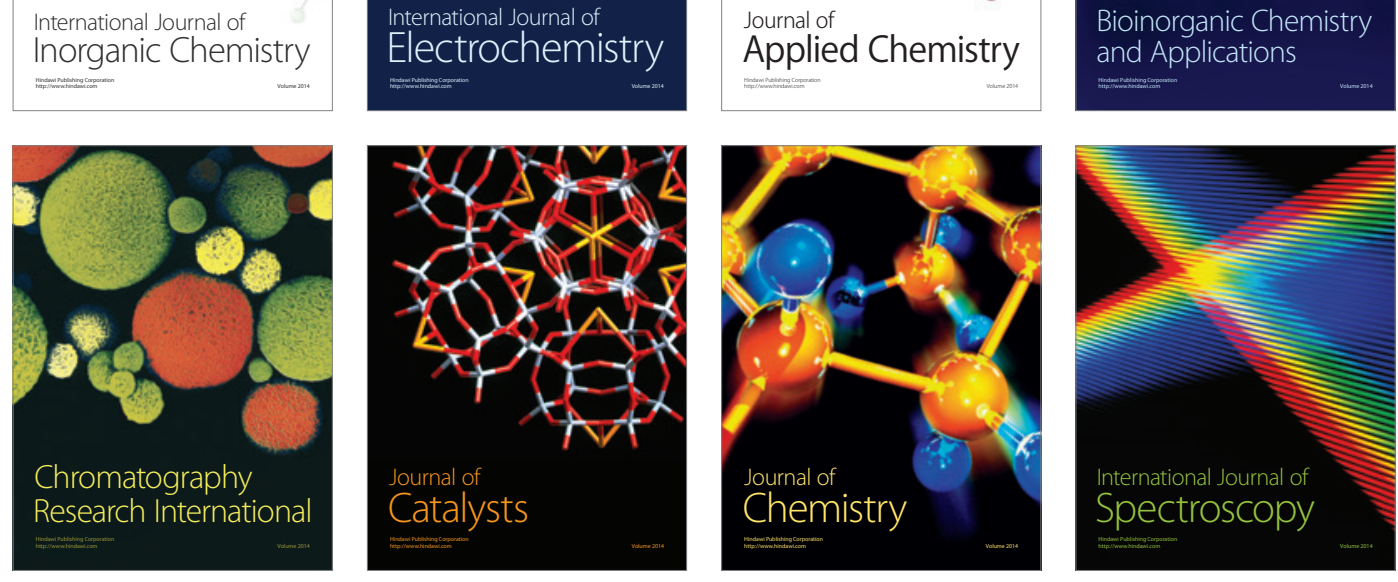